\title{
Evaluation of Auditory Working Memory Abilities in Children with Self Limited Epilepsy with Centrotemporal Spikes (SECTS): A Pilot Study
}

\author{
Anuprasad Sreenivasan ${ }^{1}$ Rajalakshmi Krishna ${ }^{2}$ Pradeep Pankajakshan Nair ${ }^{3}$ Arun Alexander ${ }^{1}$ (1) \\ ${ }^{1}$ Department of ENT, Jawaharlal Institute of Postgraduate Medical \\ Education and Research, Puducherry, India \\ Address for correspondence Anuprasad Sreenivasan, MSc, \\ Department of ENT, Jawaharlal Institute of Postgraduate Medical \\ 2 Department of Audiology, All India Institute of Speech and Hearing, \\ Education and Research, Dhanvantri Nagar, Puducherry 605006, India \\ Mysore, Karnataka, India \\ ${ }^{3}$ Department of Neurology, Jawaharlal Institute of Postgraduate \\ Medical Education and Research, Puducherry, India \\ Int J Ep 2021;7:59-63.
}

\author{
Abstract \\ Keywords \\ - self-limited epilepsy \\ with centrotemporal \\ spikes \\ - working memory \\ - digit span
}

Objectives Working memory abilities are essential in the perception of acoustic signals, especially in the presence of noise. This study aimed to measure the working memory and its associated factors in children with self-limited epilepsy with centrotemporal spikes (SECTS) and compare it with the typically developing children (TDC) without epilepsy.

Materials and Methods Twenty-two children with SECTS and 22 typically developing children participated in the study. The Digit span backward test was used for measuring working memory abilities.

Statistical Analysis and Results The working memory of children with SECTS, measured with backward digit span, was significantly poor compared with the TDC $(p<0.001)$. Factors such as the age of onset of seizures, duration of seizures, frequency of seizures and spike load did not influence the working memory.

Conclusion The children with SECTS demonstrated impairment in working memory abilities compared with normal children. The association between poor working memory and reported speech recognition impairment in children with SECTS needs to be studied.

\section{Introduction}

Self-limited epilepsy with centrotemporal spikes (SECTS) is a common focal epilepsy syndrome in childhood. ${ }^{1}$ It usually begins at the age of 3 to 13 years and disappears after puberty. ${ }^{2}$ The typical electroencephalography (EEG) findings include high voltage sharp centrotemporal spikes preceded by slow waves triggered by sleep and show a tendency to extend or change from one side to the other. ${ }^{3}$ Despite the improvements in the frequency of seizures and the remission of seizures before puberty, many children with SECTS present with disturbances in neuropsychological evaluations. ${ }^{4-6}$ Recent studies reported that children with SECTS often exhibit difficulty processing verbal messages even though their peripheral hearing sensitivity was normal. ${ }^{7}$ Over the last decades, more attention has been given to studying the influence of working memory in processing verbal messages, especially in adverse listening situations. Working memory deals with the processing and storage when performing a published online

February 21, 2022
DOI https://doi.org/ $10.1055 / \mathrm{s}-0041-1742274$. ISSN 2213-6320.

\footnotetext{
(C) 2022. Indian Epilepsy Society. All rights reserved.

This is an open access article published by Thieme under the terms of the Creative Commons Attribution-NonDerivative-NonCommercial-License, permitting copying and reproduction so long as the original work is given appropriate credit. Contents may not be used for commercial purposes, or adapted, remixed, transformed or built upon. (https://creativecommons.org/ licenses/by-nc-nd/4.0/)

Thieme Medical and Scientific Publishers Pvt. Ltd., A-12, 2nd Floor, Sector 2, Noida-201301 UP, India
} 
task. ${ }^{8,9}$ The perception of spoken language requires a listener to extract, store, and integrate rapidly varying auditory stimuli. Working memory capacity is strongly linked to the auditory perception when the signal is distorted and contributes less when the acoustic signal is not distorted. ${ }^{10}$ Most studies recommended using working memory tests when evaluating the individual's listening abilities. ${ }^{11,12}$ The studies documenting the memory abilities in children with SECTS are inconclusive and vary from no memory deficit to severe impairment. $^{13-16}$ Therefore, the present study aimed to measure the working memory abilities in children with SECTS.

\section{Materials and Methods}

\section{Participants}

This was a cross-sectional study. After the ethics committee approval, the participants were screened and recruited from the epilepsy clinic, Neurology Department, using a consecutive sampling technique. From the eligible cohort, we included children with typical clinical history and EEG findings of SECTS syndrome. ${ }^{2}$ Children with clinical characteristics of attention deficit hyperactivity disorder, autism, etc., were excluded from the study. All the participants had undergone sleep and awake EEGs. However, MRI was not done for the participants. Age- and gender-matched typically developing children (TDC) without any otological or neurological illnesses were recruited from the pediatric OPD as controls. Intelligence tests and hearing assessments were performed before the memory tests. The intelligence was screened using the extension and validated version of the Gesell drawing test (a screening IQ test). ${ }^{17}$ In this test, all participants were presented with different geometrical shapes, with the complexity of the shapes progressing from easy to difficult. The participants had to copy the shapes on an A4 size paper. Each correct response was given one point, and the incorrect response received a zero mark. The total scores were compared with the normative data, and those who passed the test were only considered for the study. Pure tone audiometry was performed for all participants using the Inventis piano dual-channel audiometer. The air conduction thresholds were tested for the octave frequencies $250 \mathrm{~Hz}$ to $8000 \mathrm{~Hz}$ using TDH 39 headphones. The bone conduction thresholds were also obtained for frequencies $250 \mathrm{~Hz}$ to $4000 \mathrm{~Hz}$. The participants with hearing sensitivity less than $15 \mathrm{~dB}$ HL were included in the study.

\section{Procedure}

We evaluated the working memory abilities using the backward digit span test by the auditory-verbal mode. The Digit span test is one of the most commonly employed tests for measuring verbal working memory. Smriti-Shravan software (version 1.0) was used for measuring the auditory digit span test. ${ }^{18}$ A laptop was used for the presentation of the stimulus. The stimuli were delivered to both ears at the most comfortable level via Sennheiser 202HD stereo headphones. In this procedure, the listeners were given sequences of numbers and asked to say the digits in the reverse order, which is displayed on the screen. After each correct response, the software increased the number of digits at one level and decreased one level to an incorrect response. There was an interstimulus interval of 500 milliseconds and a response time of 5000 milliseconds. An adaptive staircase method was employed to estimate the digit span threshold. The maximum number of digits repeated correctly was recorded.

\section{Statistical Analysis}

The statistical analysis was completed using the Statistical Package for the Social Sciences (SPSS) software version 19 (IBM Corp.; Armonk, New York). The statistical analysis included the one-sample Kolmogorov-Smirnov test for checking the normality of the distribution. Mann-Whitney $U$ test was used for comparing the digit span thresholds between children with SECTS and the TDC. The relationship between digit span thresholds and age of onset of seizures, duration of seizures, frequency of seizures and spike load were analyzed using Pearson's correlation.

\section{Results}

A total of 22 children with SECTS and 22 age- and gendermatched TDC were recruited for the study. The median (IQR) age of the children with SECTS was 10.55 (1.94) years, and of the TDC was 11.18 (1.73) years. The median age of onset of seizures was 8 years (minimum 5 , maximum 12 years). Five children had a positive family history, but information about the type of epileptic seizures in the family was not available. The demographic profile of the children with SECTS is given in -Table 1. Fifteen (68\%) children reported nocturnal seizures, one $(4.5 \%)$ had a pure daytime seizure, and the remaining six (27\%) children had a combination of the two. The total number of seizures ranged from 1 to 12 events. All children had at least one EEG characteristic of SECTS. Left centrotemporal spikes were observed in $8(36 \%)$ children, right centrotemporal spikes were seen for 4 (18\%) children, and the remaining 10 (45\%) children had bilateral discharges. Twenty participants were under anti-seizure medication, and only two of the children were not taking medications. Among the children under anti-seizure medications, most were treated with monotherapy, 19 (86\%) and only 1 (4.5\%) with multiple therapies. Sixteen (72\%) children were on sodium valproate, two $(9 \%)$ on phenytoin, and three $(13 \%)$ on carbamazepine. There was a significant difference in the backward digit span thresholds of children with SECTS compared with TDC [2.50 (0.91) versus $3.50(0.25) p<0.001$ ] ( - Table 2). The correlation between age of onset, duration, frequency of seizures and spike load with the digit span thresholds were not found to be significant ( - Table $\mathbf{3}$ ).

\section{Discussion}

The study aimed to examine the digit span as a measure of auditory working memory in children with SECTS. The study also evaluated the impact of factors such as the age of onset of seizures, duration of seizures, and the frequency of seizures on digit span thresholds. The mean digit span was 


\begin{tabular}{|c|c|c|c|c|c|c|c|c|c|c|c|c|c|c|c|c|c|c|c|c|c|c|c|}
\hline 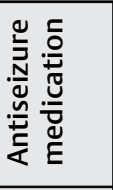 & $\stackrel{5}{\sim}$ & $\stackrel{5}{\backsim}$ & $\stackrel{\$}{\star}$ & 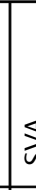 & 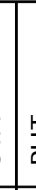 & $\frac{T}{2}$ & $\begin{array}{l}5 \\
n\end{array}$ & $\stackrel{5}{5}$ & $\stackrel{5}{n}$ & $\stackrel{5}{\sim}$ & $\stackrel{5}{n}$ & $\stackrel{5}{n}$ & z & $\stackrel{s}{n}$ & $\stackrel{5}{n}$ & $\stackrel{5}{n}$ & $\stackrel{5}{n}$ & 2 & 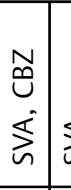 & $\stackrel{s}{n}$ & $\tilde{~}$ & జิ & 톰 \\
\hline 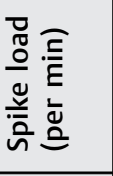 & $\stackrel{0}{-}$ & $\infty$ & 8 & s & & en & in & $m$ & $\sim$ & $\stackrel{\infty}{-}$ & $\nabla$ & 6 & in & $\nabla$ & 6 & - & $\simeq$ & $\stackrel{m}{m}$ & $\nabla$ & $\nabla$ & $\infty$, & $m$ & $\stackrel{\nabla}{\leftarrow}$ \\
\hline 空 & - & $1 \sim$ & $\tau$ & ? & & $m$ & $v$ & $m$ & $\nabla$ & $m$ & $\sim$ & $\nabla$ & $\sim$ & $\sim$ & 0 & $\stackrel{\circ}{\circ}$ & in & - & $\sim ?$ & $\simeq 1$ & $\wedge$ & $\nabla$ & - \\
\hline 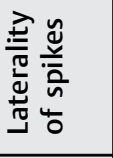 & Ғ & Еँ & $\stackrel{ \pm}{ \pm}$ & $\Phi$ & & $\begin{array}{c}0 \\
0 \\
0 \\
0\end{array}$ & $\begin{array}{c}\frac{5}{5} \\
\frac{5}{2} \\
\end{array}$ & $\stackrel{ \pm}{\Xi}$ & $\stackrel{\leftrightarrows}{\Delta}$ & \begin{tabular}{|l|} 
\\
\\
$\circ$
\end{tabular} & \begin{tabular}{|l|}
\multirow{0}{0}{} \\
$\infty$ \\
\end{tabular} & \begin{tabular}{|l|} 
\\
$\tilde{0}$ \\
$\infty$
\end{tabular} & $\begin{array}{l}\text { Fे } \\
0 \\
0\end{array}$ & 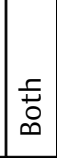 & 䓂 & $\stackrel{ \pm}{\leftrightarrows}$ & $\stackrel{ \pm}{\leftrightarrows}$ & 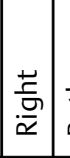 & 吾 & $\begin{array}{ll}\frac{\vec{v}}{\bar{v}} \\
\underline{\underline{v}}\end{array}$ & $\stackrel{ \pm}{\leftrightarrows}$ & $\stackrel{ \pm}{\Xi}$ & 营 \\
\hline 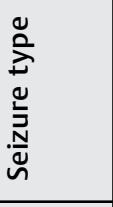 & 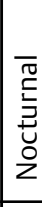 & 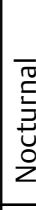 & 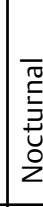 & 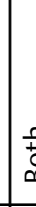 & & 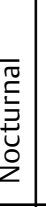 & 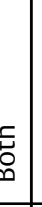 & 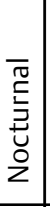 & 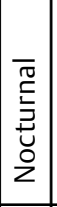 & 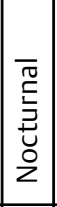 & 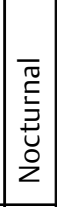 & 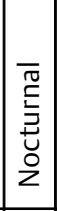 & 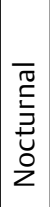 & 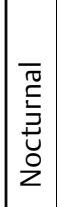 & 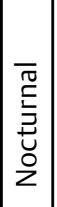 & 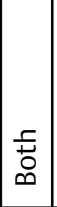 & 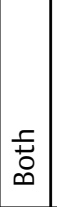 & 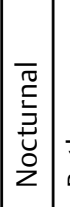 & ક્. & 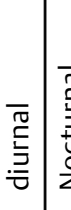 & \begin{tabular}{l|l}
$\bar{\pi}$ \\
$\stackrel{5}{5}$ \\
$\stackrel{3}{0}$ \\
$z$
\end{tabular} & 点 & 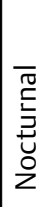 \\
\hline 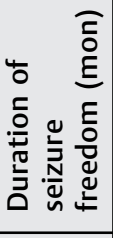 & $\stackrel{m}{m}$ & 6 & $\simeq$ & 6 & & $\simeq$ & $\underline{v}$ & $\wedge$ & in & $\simeq$ & 0 & $\stackrel{ \pm}{\sim}$ & $m$ & $\nabla$ & $\simeq$ & $\wedge$ & $\stackrel{\stackrel{\sim}{\sim}}{\sim}$ & $\sim$ & $\simeq$ & $\simeq$ & $\sim$ & $\simeq$ & $\stackrel{\simeq}{\simeq}$ \\
\hline 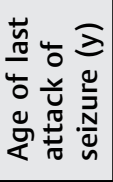 & $a$ & 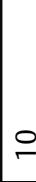 & $\stackrel{\circ}{\circ}$ & $\circ$ & & $?$ & -1 & $\infty$ & $\simeq$ & 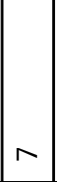 & $\circ$ & $\circ$ & $a$ & $\mp$ & $\infty$ & $\stackrel{\llcorner}{\leftarrow}$ & $\mp$ & $\infty$ & $\mp 9$ & $\therefore$ & $\circ$ & $=$ & $\simeq$ \\
\hline 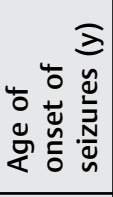 & $a$ & $\infty$ & a & 0 & & $\sigma$ & C & เn & $\simeq$ & in & $r$ & $\infty$ & $\sigma$ & 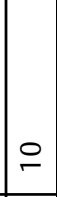 & $\wedge$ & in & $\infty$ & $\infty$ & $\stackrel{\circ}{\circ}$ & $\wedge \quad \alpha$ & $\infty$ & $\sigma$ & $\simeq$ \\
\hline 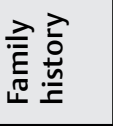 & $\stackrel{0}{2}$ & 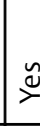 & 2 & $\frac{5}{4}$ & & : & 气 & $\stackrel{0}{z}$ & 2 & 2 & z & z & $\stackrel{\tilde{\Perp}}{\nu}$ & $\stackrel{\check{\Perp}}{\rightleftharpoons}$ & z & z & $\stackrel{0}{z}$ & $\stackrel{\Perp}{\succ}$ & 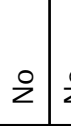 & \begin{tabular}{l|l}
$\mathbf{c}$ & $\mathbf{s}$
\end{tabular} & 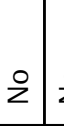 & 2 & 2 \\
\hline 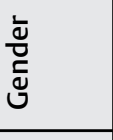 & $\frac{0}{\frac{\pi}{n}}$ & $\frac{0}{\pi}$ & 峁 & $\sum^{f}$ & & 峁 & $\frac{\frac{\pi}{2}}{20}$ & $\frac{\frac{0}{N 0}}{\sum}$ & $\frac{\frac{0}{\pi}}{\Sigma}$ & $\frac{0}{N^{\pi}}$ & \begin{tabular}{|l|}
$\frac{0}{\widetilde{T}}$ \\
$\tilde{\Xi}$ \\
$\Psi$ \\
\end{tabular} & 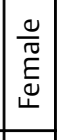 & $\begin{array}{l}\frac{0}{\pi} \\
\stackrel{0}{E} \\
\sqcup \\
\end{array}$ & 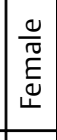 & $\frac{0}{\frac{0}{\pi}}$ & 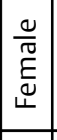 & $\frac{0}{\frac{0}{\pi}}$ & $\frac{0}{2 \pi}$ & $\frac{0}{\frac{0}{\pi}}$ & $\frac{0}{2 \pi}$ & $\frac{0}{\pi}$ & $\frac{\frac{0}{\pi}}{2 \pi}$ & $\frac{0}{\sum \pi}$ \\
\hline $\begin{array}{l}\text { ते } \\
\text { gัष }\end{array}$ & $\simeq$ & 음 & $F$ & $\circ$ & & $E$ & $\infty$ & $\infty$ & $\simeq$ & $\infty$ & $\circ$ & $\simeq$ & $a$ & $\mp$ & a & $\stackrel{\llcorner 2}{\leftarrow}$ & $\stackrel{m}{\square}$ & $\infty$ & $\simeq$ & $\mp$ & $\circ:$ & $\simeq$ & $\stackrel{m}{r}$ \\
\hline 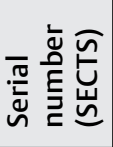 & 7 & & $n$ & & & ת & Q & $r$ & $\infty$ & $\sigma$ & 으 & $\mp$ & $\stackrel{\simeq}{\simeq}$ & $m$ & 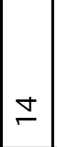 & $\stackrel{\Perp n}{\leftarrow}$ & $\stackrel{\bullet}{\circ}$ & $\approx$ & $\stackrel{\infty}{\ulcorner}$ & $\stackrel{\square}{\square}$ & $\stackrel{i}{\sim}$ & $\bar{\sim}$ & $\approx$ \\
\hline
\end{tabular}


Table 2 Comparison of digit span thresholds between SECTS and TDC

\begin{tabular}{|l|l|l|l|}
\hline Group & \multicolumn{2}{|l|}{ Median (IQR) } & -Value \\
\hline SECTS $(N=22)$ & 2.50 & 0.91 & 0.001 \\
\cline { 1 - 2 } TDC $(N=22)$ & 3.50 & 0.25 & \\
\hline
\end{tabular}

Abbreviations: N, Number of participants; $\mathrm{P}$, Statistical significance for two tailed tests.

Note: $p<0.001$ is significant. working memory abilities. This will enable the clinicians to plan early intervention programs to reduce the adverse effects of memory deficit on scholastic performance. However, our study has certain limitations. The small sample size precludes the generalization of these findings. A formal IQ test was not done for the participants. Future studies may also evaluate the association between memory abilities and understanding spoken messages in the presence of noise in children with SECTS.

Table 3 Correlation coefficient between working memory and frequency of seizures, age of onset of seizures, duration of seizures, spike load and corresponding ( $p$-Values)

\begin{tabular}{|c|c|c|c|c|c|c|c|c|}
\hline & \multicolumn{2}{|c|}{ Frequency of seizures } & \multicolumn{2}{|c|}{ Age of onset of seizures } & \multicolumn{2}{|c|}{ Duration of seizures } & \multicolumn{2}{|c|}{ Spike load } \\
\hline & $r$ & $p$-Value & $r$ & $p$-Value & $r$ & $p$-Value & $r$ & $p$-Value \\
\hline $\begin{array}{l}\text { Working } \\
\text { memory }\end{array}$ & 0.06 & 0.77 & 0.08 & 0.70 & 0.001 & 0.99 & -0.145 & 0.52 \\
\hline
\end{tabular}

Note: $p=$ Statistical significance for two tailed tests, significant $(p<0.05), r=$ correlation coefficient.

significantly lower for SECTS children than the TDC. However, the age of onset of the seizure, duration of seizures, frequency of seizures, and spike load did not influence the digit span thresholds.

The difficulties experienced by the children with SECTS in number recalling indicate a deficit in storing and manipulating the ongoing auditory input. According to the Ease of Language Understanding (ELU) model, working memory capacity is closely linked to language processing when speech is presented against background noise. The research focused on the connection between working memory and comprehending verbal messages has reported that limited working memory ability negatively affects the perception of spoken language, especially when the signal is distorted. ${ }^{19-21}$

The poor digit span threshold observed in our study was in agreement with the previous studies. ${ }^{6,22,23}$ The functional imaging studies demonstrate that cortical areas such as the left ventral and dorsal prefrontal cortex are involved in auditory perception in the presence of noise and working memory activities. ${ }^{24,25}$ The epileptiform activity can alter the function of these cortical areas. In SECTS, the epileptiform activity spreads in the neurons of the rolandic cortex covering the central fissure on both sides. ${ }^{26}$ One possibility is the spread of epileptic activity anteriorly from the rolandic cortex resulting in a functional deficit in working memory. Another explanation for this poor working memory ability is that epileptiform activities around Sylvian fissures can result in language dysfunction. This language dysfunction can affect the coding of verbal messages and leads to poor memory traces. 27,28

The present study demonstrated that children with SECTS have significant deficits in verbal working memory skills. Our findings emphasize the importance of early neuropsychological assessments, which include different measures of

\section{Conclusion}

This study evaluated the working memory abilities of children with SECTS. The results indicate that children with SECTS had poor working memory abilities compared with the TDC.

\section{Conflict of Interest}

None declared.

\section{References}

1 Wirrell EC, Camfield ES, Campfield PR. Idiopatic and benign partial epilepsies of childhood. InWyllie E, Gupta A, Lachhwani $D$, eds. The Treatment of Epilepsy. Philadelphia: Lippincott Williams \& Wilkins; 2006:373-389

2 Scheffer IE, Berkovic S, Capovilla G, et al. ILAE classification of the epilepsies: Position paper of the ILAE Commission for Classification and Terminology. Epilepsia 2017;58(04):512-521

3 Bouma PA, Bovenkerk AC, Westendorp RG, Brouwer OF. The course of benign partial epilepsy of childhood with centrotemporal spikes: a meta-analysis. Neurology 1997;48(02): 430-437

4 Baglietto MG, Battaglia FM, Nobili L, et al. Neuropsychological disorders related to interictal epileptic discharges during sleep in benign epilepsy of childhood with centrotemporal or Rolandic spikes. Dev Med Child Neurol 2001;43(06):407-412

5 Lindgren S, Kihlgren M, Melin L, Croona C, Lundberg S, EegOlofsson O. Development of cognitive functions in children with rolandic epilepsy. Epilepsy Behav 2004;5(06):903-910

6 Verrotti A, Filippini M, Matricardi S, Agostinelli MF, Gobbi G. Memory impairment and benign epilepsy with centrotemporal spike (BECTS): a growing suspicion. Brain Cogn 2014;84(01): 123-131

7 Teixeira J, Santos ME. Language skills in children with benign childhood epilepsy with centrotemporal spikes: a systematic review. Epilepsy Behav 2018;84:15-21

8 Baddeley A. The episodic buffer: a new component of working memory? Trends Cogn Sci 2000;4(11):417-423 
9 Miyake A, Shah P, eds. 1999. Models of Working Memory. New York: Cambridge University Press

10 Rönnberg J, Rudner M, Lunner T, Zekveld AA. When cognition kicks in: working memory and speech understanding in noise. Noise Health 2010;12(49):263-269

11 Conway AR, Cowan N, Bunting MF. The cocktail party phenomenon revisited: the importance of working memory capacity. Psychon Bull Rev 2001;8(02):331-335

12 Meister H, Rählmann S, Lemke U, Besser J. Verbal response times as a potential indicator of cognitive load during conventional speech audiometry with matrix sentences. Trends Hear 2018; 22:2331216518793255

13 D’Alessandro P, Piccirilli M, Tiacci C, et al. Neuropsychological features of benign partial epilepsy in children. Ital J Neurol Sci 1990;11(03):265-269

14 Croona C, Kihlgren M, Lundberg S, Eeg-Olofsson O, Eeg-Olofsson KE. Neuropsychological findings in children with benign childhood epilepsy with centrotemporal spikes. Dev Med Child Neurol 1999;41(12):813-818

15 Danielsson J, Petermann F. Cognitive deficits in children with benign rolandic epilepsy of childhood or rolandic discharges: a study of children between 4 and 7 years of age with and without seizures compared with healthy controls. Epilepsy Behav 2009;16 (04):646-651

16 Northcott E, Connolly AM, Berroya A, et al. The neuropsychological and language profile of children with benign rolandic epilepsy. Epilepsia 2005;46(06):924-930

17 Srinivasan V. Extension and validation of Gessel's drawing test of intelligence in a group of children with communicating handicaps. Indian J Clin Psychol 2002;29(02):123-127

18 Kumar UA, Sandeep M. Development and test trail of computer based auditory-cognitive training module for individuals with cochlear hearing loss. Unpublished departmental project. Mysuru AIISH; 2013
19 Rosen S, Cohen M, Vanniasegaram I. Auditory and cognitive abilities of children suspected of auditory processing disorder (APD). Int J Pediatr Otorhinolaryngol 2010;74(06):594-600

20 Besser J, Koelewijn T, Zekveld AA, Kramer SE, Festen JM. How linguistic closure and verbal working memory relate to speech recognition in noise-a review. Trends Amplif 2013;17(02):75-93

21 Souza P, Arehart K, Neher T. Working memory and hearing aid processing: Literature findings, future directions, and clinical applications. Front Psychol 2015;6:1894

22 Parisi P, Matricardi S, Tozzi E, Sechi E, Martini C, Verrotti A. Benign epilepsy of childhood with centro-temporal spikes (BECTS) versus migraine: a neuropsychological assessment. Childs Nerv Syst 2012;28(12):2129-2135

23 Deltour L, Quaglino V, Barathon M, De Broca A, Berquin P. Clinical evaluation of attentional processes in children with benign childhood epilepsy with centrotemporal spikes (BCECTS). Epileptic Disord 2007;9(04):424-431

24 Wong PCM, Ettlinger M, Sheppard JP, Gunasekera GM, Dhar S. Neuroanatomical characteristics and speech perception in noise in older adults. Ear Hear 2010;31(04):471-479

25 Wong PCM, Jin JX, Gunasekera GM, Abel R, Lee ER, Dhar S. Aging and cortical mechanisms of speech perception in noise. Neuropsychologia 2009;47(03):693-703

26 Panayiotopoulos CP, Michael M, Sanders S, Valeta T, Koutroumanidis M. Benign childhood focal epilepsies: assessment of established and newly recognized syndromes. Brain 2008;131(Pt 9):2264-2286

27 Bulgheroni S, Franceschetti S, Vago C, et al. Verbal dichotic listening performance and its relationship with EEG features in benign childhood epilepsy with centrotemporal spikes. Epilepsy Res 2008;79(01):31-38

28 Metz-Lutz MN, Filippini M. Neuropsychological findings in Rolandic epilepsy and Landau-Kleffner syndrome. Epilepsia 2006;47 (Suppl 2):71-75 